\title{
Ptosis in systemic lupus erythematosus
}

\author{
JOHN G. LANHAM \\ M.R.C.P.
}

\author{
KEITH B. ELKON \\ M.R.C.P.
}

\author{
GRAHAM R. V. HUGHES \\ M.D., F.R.C.P.
} Rheumatology Unit, Department of Medicine, Hammersmith Hospital and Royal Postgraduate Medical School,
Du Cane Road, London W12

\section{Summary}

Mild unilateral ptosis was observed in 11 women with systemic lupus erythematosus. It was associated with active disease in 8 patients, but only 2 had overt cerebral involvement. Isolated ptosis should not therefore be regarded as an indication of cerebral lupus.

\section{Introduction}

Ptosis is only rarely mentioned as a manifestation of systemic lupus erythematosus (SLE) and is generally interpreted as an indication of cerebral disease (Harvey et al., 1954; Grigor et al., 1978; Feinglass et al., 1976). As we had observed ptosis in the absence of cerebral lupus, we reviewed the records of all SLE patients with ptosis seen in our unit over the past six years in order to ascertain the clinical significance of this phenomenon.

\section{Patients: methods and results}

Eleven women were found to have unilateral ptosis associated with SLE; all patients satisfied the preliminary American Rheumatism Association criteria for the classification of the disease (Cohen et al., 1971). Ptosis was diagnosed if the eyelid overlapped the upper edge of the pupil (Fig. 1). It was mild in most patients, but obvious enough for all the women to have recognized the phenomenon themselves; it was not severe enough in any to obscure vision. As approximately 400 new patients with SLE were seen in this unit in the 6-year period the prevalence of ptosis in our SLE population is about $3 \%$.

The SLE was assessed as being active if one or more of the following features were present: skin rash, arthritis, nephritis, pericarditis, cerebral disease, myositis or fever. Cerebral disease was diagnosed if focal neurological signs, convulsions, psychosis or an organic brain syndrome occurred.

Of the 7 patients with intermittent ptosis, 3 had episodes lasting only a few days, while the other 4 had episodes of several weeks duration. Two patients had ptosis only once and in both of these women it persisted for about a month. Two patients had constant ptosis, which increased in severity in one whenever her SLE was active. Similarly, 7 other women had ptosis which appeared or worsened during disease exacerbations, but in only 2 of these individuals was evidence of cerebral disease activity related to the ptosis. Therefore 8 of the 11 patients developed ptosis in the context of active disease.

\section{Discussion}

The pathogenesis of ptosis in SLE is unclear, but probably more than one mechanism is implicated. We found no evidence of myasthenia gravis in our patients (two had negative Tensilon tests) and in only one woman was an overt cranial nerve palsy detected to account for the phenomenon. She had a retrobulbar headache and an oculomotor nerve palsy of three weeks duration. Apart from a second patient with retrobulbar headaches, none of the others had ocular symptoms or signs. Only one patient had a positive family history of ptosis; this woman had a mother with mild unilateral ptosis, but ptosis only developed in her after the onset of SLE.

It is uncertain whether ptosis in SLE is neurogenic or myogenic in origin, but there is little evidence to support the contention that isolated ptosis indicates cerebral lupus. We offer our impression that ptosis, especially mild unilateral ptosis, is a not uncommon feature of SLE, and generally occurs while the disease is active. 


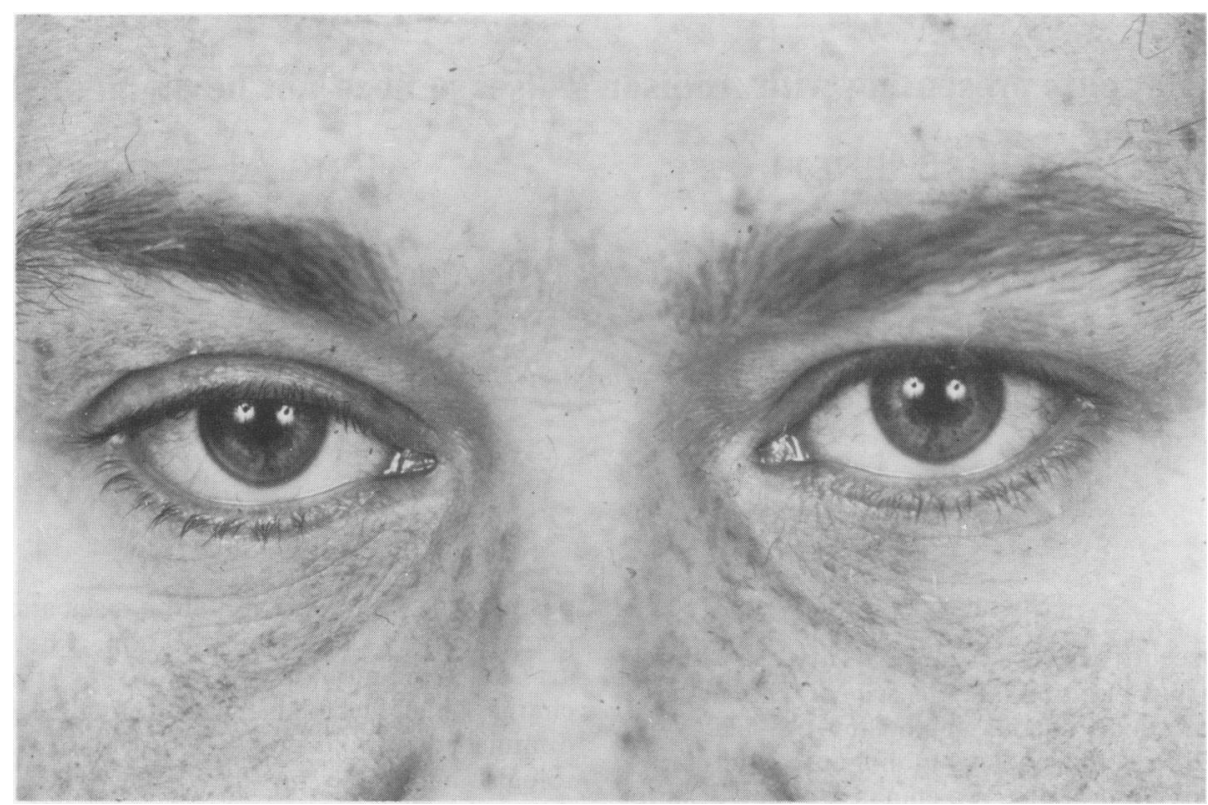

FIG. 1. Photograph showing mild right sided ptosis in a patient with systemic lupus erythematosus.

\section{References}

Cohen, A.S., Reynolds, W.E., Franklin, E.C., Kulka, J.P., Ropes, M.L., Shulman, L.E. \& Wallace, S.N. (1971) Preliminary criteria for the classification of systemic lupus erythematosus. Bulletin on Rheumatic Diseases, 21, 643.

Feinglass, E.J., ARNeTt, F.C., DorsCh, C.A., Zizic, T.M. \& STEVENS, M.B. (1976) Neuropsychiatric manifestations of systemic lupus erythematosus: diagnosis, clinical spectrum and relationship to other features of the disease. Medicine (Baltimore), 54, 323.

Grigor, R.R., Edmonds, J., LewKonia, R., Bresnihan, B. \& HuGHES, G.R.V. (1978) Systemic lupus erythematosus: a prospective analysis. Annals of Rheumatic Diseases, 37, 121.

Harvey, A., Shulman, L.E., Tumulty, P.A., Couley, C.L. \& SCHOENRICH, E.M. (1954) Systemic lupus erythematosus. Review of the literature and clinical analysis of 138 cases. Medicine (Baltimore), 33, 291. 